\title{
A New Framework for the Determination of the Eigenvalues and Eigenfunctions of the Quantum Harmonic Oscillator
}

\author{
Francis T. Oduro ${ }^{1,2}$, Amos Odoom ${ }^{1}$ \\ ${ }^{1}$ Department of Mathematics, Kwame Nkrumah University of Science and Technology (KNUST), Kumasi, Ghana \\ ${ }^{2}$ African Institute of Mathematical Sciences (AIMS), Accra, Ghana \\ Correspondence: Francis T. Oduro, Department of Mathematics, Kwame Nkrumah University of Science and Technology \\ (KNUST), Kumasi, Ghana
}

Received: September 5, 2021 Accepted: October 14, 2021 Online Published: October 27, 2021

doi:10.5539/jmr.v13n6p20

URL: https://doi.org/10.5539/jmr.v13n6p20

\begin{abstract}
This study was designed to obtain the energy eigenvalues and the corresponding Eigenfunctions of the Quantum Harmonic oscillator through an alternative approach. Starting with an appropriate family of solutions to a relevant linear differential equation, we recover the Schrödinger Equation together with its eigenvalues and eigenfunctions of the Quantum Harmonic Oscillator via the use of Gram Schmidt orthogonalization process in the usual Hilbert space. Significantly, it was found that there exists two separate sequences arising from the Gram Schmidt Orthogonalization process; one in respect of the even eigenfunctions and the other in respect of the odd eigenfunctions.
\end{abstract}

Keywords: quantization, linear harmonic oscillator, orthogonalization

\section{Introduction}

The Quantum Harmonic Oscillator is perhaps the most celebrated and useful of all the Quantum Mechanical systems with applications in all areas of modern physics from subatomic and molecular physics through condensed matter physics to high energy physics and to more exotic fields such as Quantum Computing and Quantum Finance (Wolf, 2021 \& Schaden, 2002).

In the context of the standard formulation of Quantum Mechanics, Schrödinger Equation is obtained from the classical energy equation

$$
E=\frac{1}{2} \frac{p^{2}}{2 m}+\frac{1}{2} m \omega^{2} x^{2}
$$

in which the system can be put in Quantum form (Quantization) by means of the correspondence principle where we have the associations:

$$
\begin{array}{r}
\hat{x} \rightarrow x \\
\hat{p} \rightarrow-\frac{i \hbar \partial}{\partial x} \\
\hat{E} \rightarrow \frac{i \hbar \partial}{\partial t}
\end{array}
$$

The Hamiltonian of the Harmonic Oscillator is an operator which acting on a wave function $\Psi_{n}$ is given by

$$
H_{n} \Psi_{n}=-\frac{\hbar^{2}}{2 m} \frac{d^{2} \Psi_{n}}{d x^{2}}+\frac{1}{2} m \omega^{2} x^{2} \Psi_{n}
$$

where $m, p, \omega, \hbar$ have their usual meanings. In this paper, without loss of generality, we shall use standardized units such that $m=\omega=\hbar=1$. (Bransden,2000).

Existing approaches of solving the Schrödinger Equation for the eigenvalues and eigenfunctions among others are the Ladder Operator Method and Series method.

A new framework is proposed in this paper which deviates slightly from the above approach which uses the correspondence principle directly. While we do not abandon the usual assumptions of Quantum Mechanics and its pertinent mathematical framework, we rather proceed as follows: Starting with appropriate solutions of a relevant Second Order linear 
differential equation, we seek to recover the Schrödinger Equation of the Quantum Harmonic Oscillator together with its eigenvalues and eigenfunctions.

Our motivation is to eventually formulate an effective framework for studying the Quantum Anharmonic oscillator. This study on Quantum Anharmonic Oscillator, we hope to do in a subsequent paper. The structure of the current paper is as follows: We provide in the next section a very brief review of the mathematical foundations of Quantum Mechanics restating some relevant theorems. This is followed by a section describing the proposed method in terms of a few propositions which are proved. In subsequent sections, we carry out explicit computations, based on our method, of the eigenvalues and eigenfunctions of the Quantum Harmonic oscillator for even and odd cases.

\section{Brief Theory}

\subsection{Postulates of Quantum Mechanics}

To an ensemble of physical systems one can, in certain cases associate a wave function or state function which contains all the information that can be known about the ensemble. This function is in general complex; it may be multiplied by an arbitrary complex number altering its physical significance. A wave function $\Psi(x, t)$ is said to be square integrable if its normalization integral $I=\int|\Psi(r, t)|^{2} d r$ is finite, where the integration is over the real line. It also means that the quantity $P(x, t)=|\Psi(x, t)|^{2}$ is interpreted as the Position probability density - Born's Rule (Bransden, 2000).

The state of a system is represented by a normalized vector $(|\Psi\rangle)$ in the $L_{2}$ (Hilbert ). There is an inner product denoted by, $\langle\Psi \mid \Phi\rangle$ for any two elements in the space. For example, for functions defined in the interval $a \leq x \leq b$ (in one dimension), we may write

$$
<\Psi \mid \Phi>=\int_{a}^{b} \Phi^{*} \Psi d x
$$

Observable quantities $Q(x, p, t)$ are represented by Hermitian operators $\hat{Q}\left(x, \frac{\hbar}{i}, \frac{\partial}{\partial x}, t\right)$; the expectation value of $Q$ in the state $|\Psi\rangle$, is $\langle\Psi \mid \hat{Q} \Psi\rangle$. A measurement of the observable $Q$ on a particle in the state $|\Psi\rangle$ is certain to retain the value $\lambda$ if and only if $|\Psi\rangle$ is an eigenfunction of $\hat{Q}$, with eigenvalue $\lambda$. For example, the time independent Schrödinger equation $\hat{H} \Psi=E \Psi$ is the eigenvalue equation for the Hamiltonian operator. The eigenfunction corresponds to a state in which the total energy has a precise numerical value $E$. (Griffiths,1999)

The Superposition Principle states that if the state function $\Psi_{1}$ is associated with one possible state of a statistical ensemble of physical systems, and the state function $\Psi_{2}$ with another state of this ensemble, then any linear combination

$$
\Psi=c_{1} \Psi_{1}+c_{2} \Psi_{2}
$$

where $c_{1}$ and $c_{2}$ are complex constants, is also a state function associated with a possible state of the ensemble.

With every dynamical variable is associated a linear operator. That is, an operator $\hat{A}$ is linear if it has the property

$$
\hat{A}\left(c_{1} \Psi_{1}+c_{2} \Psi_{2}\right)=c_{1}\left(\hat{A} \Psi_{1}\right)+c_{2}\left(\hat{A} \Psi_{2}\right)
$$

where $\Psi_{1}$ and $\Psi_{2}$ are two functions and $c_{1}$ and $c_{2}$ are complex constants.

The only result of a precise measurement of the dynamical variable $A$ is one of the eigenvalue $\lambda$ of the linear operator $\hat{A}$ associated with $A$. The linear operators required are called Hermitian operators and are defined by the condition

$$
\langle\Psi \mid(\hat{A} \Psi)\rangle=\langle(\hat{A} \Psi) \mid \Psi\rangle
$$

where $\Psi$ is a square integrable function. If $\Psi$ is an eigenfunction of the operator $\hat{A}$ corresponding to the eigenvalue $\lambda$, then we have

$$
\left\langle\Psi_{n}|\hat{A}| \Psi_{n}\right\rangle=\lambda_{n}\left\langle\Psi_{n} \mid \Psi_{n}\right\rangle
$$

In addition, since

$$
\left(\hat{A} \Psi_{n}\right)^{*}=\lambda_{n}^{*} \Psi_{n}^{*}
$$

we also have

$$
\left\langle\left(A \Psi_{n}\right) \mid \Psi_{n}\right\rangle=\lambda_{n}^{*}\left\langle\Psi_{n} \mid \Psi_{n}\right\rangle
$$

If $\hat{A}$ is Hermitian, then the left-hand sides of equations (7) and (8) are equal, and hence $\lambda_{n}=\lambda_{n}^{*}$.

In another vein, two different eigenfunctions belonging to the same eigenvalue are orthogonal. (Bransden, 2000) 


\subsubsection{Gram Schmidt Orthogonalization Process}

Suppose $\left\{U_{1}, U_{2} \ldots U_{n}\right\}$ is a basis of an inner product space $V$. One can use this basis to construct an orthogonal basis $\left\{V_{1}, V_{2}, \ldots V_{n}\right\}$ of $V$ as follows.

Set

$$
\begin{gathered}
V_{1}=U_{1} \\
V_{2}=U_{2}-\frac{\left\langle U_{2}, V_{1}\right\rangle}{\left\langle V_{1}, V_{1}\right\rangle} V_{1} \\
V_{3}=U_{3}-\frac{\left\langle U_{3}, V_{1}\right\rangle}{\left\langle V_{1}, V_{1}\right\rangle} V_{1}-\frac{\left\langle U_{3}, V_{2}\right\rangle}{\left\langle V_{2}, V_{2}\right\rangle} V_{2} \\
V_{4}=U_{4}-\frac{\left\langle U_{4}, V_{1}\right\rangle}{\left\langle V_{1}, V_{1}\right\rangle} V_{1}-\frac{\left\langle U_{4}, V_{2}\right\rangle}{\left\langle V_{2}, V_{2}\right\rangle} V_{2}-\frac{\left\langle U_{4}, V_{3}\right\rangle}{\left\langle V_{3}, V_{3}\right\rangle} V_{3}
\end{gathered}
$$

In general, for $V_{n}$, we have

$$
V_{n}=U_{n}-\frac{\left\langle U_{n}, V_{1}\right\rangle}{\left\langle V_{1}, V_{1}\right\rangle} V_{1}-\frac{\left\langle U_{n}, V_{2}\right\rangle}{\left\langle V_{2}, V_{2}\right\rangle} V_{2}-\ldots-\frac{\left\langle U_{n}, V_{n-1}\right\rangle}{\left\langle V_{n-1}, V_{n-1}\right\rangle} V_{n-1}
$$

where the symbol $\langle\ldots, \ldots\rangle$ in section 2.1.1 above represents the inner product between of two eigenfunctions. (Miller, 2008)

\section{Results and Discussion}

We seek wave functions that reflect the symmetry of the simple harmonic motion. In addition, these functions are to be smooth and must at the very least be a solution pair (by virtue of Born's Rule) of some Second Order Linear Differential Equation. Thus, the most basic of such a pair would be the functions $e^{-\frac{x^{2}}{2}}$ and $x e^{-\frac{x^{2}}{2}}$ in order to ensure a non - vanishing Wronskian (Spiegel, 1976). These ideas are what motivate the next results.

\subsection{Theoretical Results}

\subsubsection{Proposition 1}

The family of functions

$$
U_{n}=x^{n} e^{-\frac{x^{2}}{2}}
$$

where $n=0,1,2, \ldots$ constitutes solutions to the following second order linear differential equation

$$
U_{n}^{\prime \prime}=n(n-1) x^{-2} U_{n}-(2 n+1) U_{n}+x^{2} U_{n}
$$

\section{Proof}

We apply Leibniz Theorem to equation (14). Now, let $U_{n}=u v$ where $u=x^{n}$ and $v=e^{-\frac{x^{2}}{2}}$. If $u$ and $v$ are smooth functions, and we substitute the results into Leibniz expansion $U_{n}^{\prime \prime}=u^{\prime \prime} v+2 u^{\prime} v^{\prime}+u v^{\prime \prime}$ (Mendelson,2004), we generate equation (15).

\section{Example}

Inserting $n=0$ and $n=1$ into equation (15), we get the following

$$
\begin{aligned}
& {\left[-\frac{1}{2} \frac{d^{2}}{d x^{2}}+\frac{1}{2} x^{2}\right] U_{0}(x)=\frac{1}{2} U_{0}(x)} \\
& {\left[-\frac{1}{2} \frac{d^{2}}{d x^{2}}+\frac{1}{2} x^{2}\right] U_{1}(x)=\frac{3}{2} U_{1}(x)}
\end{aligned}
$$

Equations (16) and (17) are eigenvalue problems with eigenvalues $\frac{1}{2}$ and $\frac{3}{2}$ respectively, and their corresponding eigenfunctions $U_{0}=e^{-\frac{x^{2}}{2}}$ and $U_{1}=x e^{-\frac{x^{2}}{2}}$. The two (Schrodinger) equations represent respectively the ground state and the first excited state to the Schrödinger equation respectively. 


\section{Remark 1}

However, for $n \geq 2$, we failed to obtain an eigenvalue problem and therefore we are not able to immediately recover the Schrödinger Equation.

For instance, for $n=2,3$, we have

$$
\begin{aligned}
& {\left[-\frac{1}{2} \frac{d^{2}}{d x^{2}}+\frac{1}{2} x^{2}\right] U_{2}(x)=\frac{5}{2} U_{2}(x)+U_{0}(x)} \\
& {\left[-\frac{1}{2} \frac{d^{2}}{d x^{2}}+\frac{1}{2} x^{2}\right] U_{3}(x)=\frac{7}{2} U_{3}(x)+3 U_{1}(x)}
\end{aligned}
$$

Equations (18) and (19) have extra terms such as $U_{0}(x)$ and $3 U_{1}(x)$ respectively.

\subsubsection{Proposition 2}

There exists a mutually orthogonal family of functions $V_{m}=\sum_{k=0}^{m} C_{k} U_{k}$ which constitutes a solution to the equation

$$
\left[-\frac{1}{2} \frac{d^{2}}{d x^{2}}+\frac{1}{2} x^{2}\right] V_{m}=\left(m+\frac{1}{2}\right) V_{m} .
$$

where $U_{k}$ belong to the family of functions given by equation (14)

\section{Proof}

Equation (20) is an eigenvalue problem involving a Hermitian operator and the solutions of this equation are therefore mutually orthogonal (See section 2). It is clear that to convert equation (15) to equation (20), we need to take linear combinations of the family $U_{k}$ which will solve equation (15) such that the first term of equation (15) vanishes. Such Linear combinations can be obtained through Gram Schmidt Orthogonalization process. Thus,

$$
V_{m}=U_{m}-\sum_{k=0,1}^{m-2} \frac{\left\langle U_{m}, V_{k}\right\rangle}{\left\langle V_{k}, V_{k}\right\rangle} V_{k}
$$

For eigenfunctions with $m$ even,i.e, $m=0,2,4,6, .$. , and $m$ odd, i.e. $m=1,3,5, \ldots$, where the initializations are respectively given by $V_{0}=U_{0}$ and $V_{1}=U_{1}$.

\section{Remark 2}

The family of functions $U_{n}=x^{n} e^{-\frac{x^{2}}{2}}$ are alternately even or odd functions. Since even and odd functions are already orthogonal, it follows that we cannot apply Gram Schmidt Orthogonalization process to consecutive functions in the sequence. This is because consecutive functions are already orthogonal. Hence, we can only apply the Gram Schmidt Orthogonalization process to alternate members of the sequence that are only even functions or only odd functions.

\section{Some Examples}

In reference to proposition 2 , if we substitute $m=2,3, \ldots$ into equation (20), we will get

$$
\begin{aligned}
& {\left[-\frac{1}{2} \frac{d^{2}}{d x^{2}}+\frac{1}{2} x^{2}\right] V_{2}=\frac{5}{2} V_{2}} \\
& {\left[-\frac{1}{2} \frac{d^{2}}{d x^{2}}+\frac{1}{2} x^{2}\right] V_{3}=\frac{7}{2} V_{3}}
\end{aligned}
$$

\section{Remark 3}

It is noteworthy that equations (22) give the well known eigenvalues of the Quantum Harmonic Oscillator. However , we still need to compute the corresponding eigenfunctions.

\subsection{Specific Computations of the Eigenfunctions of the Quantum Harmonic Oscillator}

\subsubsection{Even Eigenfunctions for $m \geq 2$}

According to the Proposition 2 , in order to get the eigenvalue problem, we need to apply Gram Schmidt Orthogonalization process to the family of solutions $U_{m}$ for $m \geq 2$ in order also to compute the eigenfunctions.

For the case $m=2$ we start with the initialization condition by setting

$$
V_{0}=U_{0}=e^{-\frac{x^{2}}{2}}
$$


We again have

$$
U_{2}=x^{2} e^{-\frac{x^{2}}{2}}
$$

Applying the Gram Schmidt equation, we have

$$
V_{2}=x^{2} e^{-\frac{x^{2}}{2}}-\frac{\left\langle x^{2} e^{-\frac{x^{2}}{2}}, e^{-\frac{x^{2}}{2}}\right\rangle}{\left\langle e^{-\frac{x^{2}}{2}}, e^{-\frac{x^{2}}{2}}\right\rangle} U_{0}
$$

This is expanded as

$$
V_{2}=x^{2} e^{-\frac{x^{2}}{2}}-\frac{\int_{-\infty}^{+\infty} x^{2} e^{-x^{2}} d x}{\int_{-\infty}^{+\infty} e^{-x^{2}} d x} e^{-\frac{x^{2}}{2}}
$$

Equation (26) may be simplified as

$$
V_{2}=x^{2} e^{-\frac{x^{2}}{2}}-\frac{\frac{\sqrt{\pi}}{2}}{\sqrt{\pi}} e^{-\frac{x^{2}}{2}}=\left(x^{2}-\frac{1}{2}\right) e^{-\frac{x^{2}}{2}}
$$

Similarly, we want to generate $V_{4}$ as we substitute $V_{0}, V_{2}, U_{2}$, and $U_{4}$ into the Gram Schmidt equation. This gives us

$$
\begin{array}{r}
V_{4}=x^{4} e^{-\frac{x^{2}}{2}}-\frac{\left\langle x^{4} e^{-\frac{x^{2}}{2}}, e^{-\frac{x^{2}}{2}}\right\rangle}{\left\langle e^{-\frac{x^{2}}{2}}, e^{-\frac{x^{2}}{2}}\right\rangle} e^{-\frac{x^{2}}{2}} \\
-\frac{\left\langle x^{4} e^{-\frac{x^{2}}{2}},\left(x^{2}-\frac{1}{2}\right) e^{-\frac{x^{2}}{2}}\right\rangle}{\left\langle\left(x^{2}-\frac{1}{2}\right) e^{-\frac{x^{2}}{2}},\left(x^{2}-\frac{1}{2}\right) e^{-\frac{x^{2}}{2}}\right\rangle}\left(x^{2}-\frac{1}{2}\right) e^{-\frac{x^{2}}{2}}
\end{array}
$$

Equation (28) is simplified as

$$
\begin{gathered}
V_{4}=x^{4} e^{-\frac{x^{2}}{2}}-\frac{\int_{-\infty}^{+\infty} x^{4} e^{-x^{2}} d x}{\int_{-\infty}^{+\infty} e^{-x^{2}} d x} e^{-\frac{x^{2}}{2}} \\
-\frac{\int_{-\infty}^{+\infty}\left(x^{2}-\frac{1}{2}\right) x^{4} e^{-x^{2}} d x}{\int_{-\infty}^{+\infty}\left(x^{2}-\frac{1}{2}\right)^{2} e^{-x^{2}} d x}\left(x^{2}-\frac{1}{2}\right) e^{-\frac{x^{2}}{2}} \\
\Longrightarrow V_{4}=x^{4} e^{-\frac{x^{2}}{2}}-\frac{\frac{3 \sqrt{\pi}}{4}}{\sqrt{\pi}} e^{-\frac{x^{2}}{2}}-\frac{\frac{3}{2} \sqrt{\pi}}{\frac{\sqrt{\pi}}{2}}\left(x^{2}-\frac{1}{2}\right) e^{-\frac{x^{2}}{2}} \\
V_{4}=\left(x^{4}-3 x^{2}+\frac{3}{4}\right) e^{-\frac{x^{2}}{2}}
\end{gathered}
$$

Therefore, the orthogonal basis $\left\{V_{0}, V_{2}, V_{4}\right\}$ are written as

$$
\left\{e^{-\frac{x^{2}}{2}},\left(x^{2}-\frac{1}{2}\right) e^{-\frac{x^{2}}{2}},\left(x^{4}-3 x^{2}+\frac{3}{4}\right) e^{-\frac{x^{2}}{2}}, \ldots\right\}
$$

Equation (31) replaces the original trial solution basis $\left\{U_{0}, U_{2}, U_{4}\right\}$. Thus, equation (31) gives the orthogonal basis corresponding to even functions which were orthogonalized.

3.2.2 Verification that the Even Eigenfunctions $V_{2}$ and $V_{4}$ are Solutions of the Schrödinger Equation

Consider

$$
V_{2}=\left(x^{2}-\frac{1}{2}\right) e^{-\frac{x^{2}}{2}}
$$

Substituting (32) into equation (15) and applying Leibniz's Theorem, we obtain

$$
\begin{aligned}
& V_{2}^{\prime \prime}=2 e^{-\frac{x^{2}}{2}}+2(2 x)\left(-x e^{-\frac{x^{2}}{2}}\right)+\left(x^{2}-\frac{1}{2}\right)\left(x^{2}-1\right) e^{-\frac{x^{2}}{2}} \\
& \Longrightarrow V_{2}^{\prime \prime}=-4\left(x^{2}-\frac{1}{2}\right) x^{2} e^{-\frac{x^{2}}{2}}+\left(x^{2}-\frac{1}{2}\right)\left(x^{2}-1\right) e^{-\frac{x^{2}}{2}}
\end{aligned}
$$


Equation (33) may finally be simplified and written as

$$
\left[-\frac{1}{2} \frac{d^{2}}{d x^{2}}+\frac{1}{2} x^{2}\right] V_{2}=\frac{5}{2} V_{2}
$$

Similarly, we want to consider

$$
V_{4}=\left(x^{4}-3 x^{2}+\frac{3}{4}\right) e^{-\frac{x^{2}}{2}}
$$

Applying Leibniz expansion to equation (35), it is simplified as

$$
V_{4}^{\prime \prime}=-8\left(x^{4}-3 x^{2}+\frac{3}{4}\right) e^{-\frac{x^{2}}{2}}+\left(x^{4}-3 x^{2}+\frac{3}{4}\right)\left(x^{2}-1\right) e^{-\frac{x^{2}}{2}}
$$

If we substitute (35) into equation (36), we can simplify it as

$$
V_{4}^{\prime \prime}=-8 V_{4}+x^{2} V_{4}-V_{4}
$$

Equation (37) can finally be written as

$$
\left[-\frac{1}{2} \frac{d^{2}}{d x^{2}}+\frac{1}{2} x^{2}\right] V_{4}=\frac{9}{2} V_{4}
$$

Therefore, equations (34) and (38) represent the precise (Schrodinger) equations for the quantum harmonic oscillator with the eigenfunctions $V_{2}$ and $V_{4}$ respectively.

3.2.3 Odd Eigenfunctions of the Quantum Harmonic Oscillator for $m \geq 3$

Similarly, we want to consider the Proposition 2 given above,and apply Gram Schmidt

Orthogonalization process to the odd trial solutions for $m \geq 3$ in order to generate the Schrödinger equations.

For convenience, we want to find $V_{3}$.

We now start by inserting $n=1$ and $n=3$ into equation (14) and this gives

$$
\begin{gathered}
V_{1}=U_{1}=x e^{-\frac{x^{2}}{2}} \\
U_{3}=x^{3} e^{-\frac{x^{2}}{2}}
\end{gathered}
$$

Substituting $V_{1}$ and $U_{3}$ into the Gram Schmidt equation and proceeding as in the even case, we get

$$
\begin{gathered}
V_{3}=x^{3} e^{-\frac{x^{2}}{2}}-\frac{\int_{-\infty}^{+\infty} x^{4} e^{-x^{2}} d x}{\int_{-\infty}^{+\infty} x^{2} e^{-x^{2}} d x} x e^{-\frac{x^{2}}{2}} \\
V_{3}=x^{3} e^{-\frac{x^{2}}{2}}-\frac{\frac{3 \sqrt{\pi}}{4}}{\frac{\sqrt{\pi}}{2}} x e^{-\frac{x^{2}}{2}}=\left(x^{2}-\frac{3}{2}\right) x e^{\frac{x^{2}}{2}}
\end{gathered}
$$

We can go through the same process to generate $V_{5}$ as

$$
V_{5}=\left(x^{4}-5 x^{2}+\frac{15}{4}\right) x e^{-\frac{x^{2}}{2}}
$$

The Orthogonal Basis are

$$
\left\{x e^{-\frac{x^{2}}{2}},\left(x^{2}-\frac{3}{2}\right) x e^{-\frac{x^{2}}{2}},\left(x^{4}-5 x^{2}+\frac{15}{4}\right) x e^{-\frac{x^{2}}{2}}\right\}
$$

3.2.4 Verification that the Odd Eigenfunctions $V_{3}$ and $V_{5}$ are Solutions of the Schrödinger Equation

Proceeding in the same way as in the even case, the Schrödinger Equation for the eigenfunction $V_{3}$ and $V_{5}$ when calculated are given by

$$
\left[-\frac{1}{2} \frac{d^{2}}{d x^{2}}+\frac{1}{2} x^{2}\right] V_{3}=\frac{7}{2} V_{3}
$$




$$
\left[-\frac{1}{2} \frac{d^{2}}{d x^{2}}+\frac{1}{2} x^{2}\right] V_{5}=\frac{11}{2} V_{5}
$$

Equations (45) and (46) are also eigenvalue equations which have exact eigenvalues as compared with the Linear Harmonic Oscillator.

Table 1. A summary of the results obtained in the sample calculations in section 3

\begin{tabular}{ccc}
\hline & $\begin{array}{c}\text { Eigenvalues, } \\
\left(m+\frac{1}{2}\right)\end{array}$ & $\begin{array}{c}\text { Eigenfunctions, } \\
V_{m}\end{array}$ \\
\hline 0 & $\frac{1}{2}$ & $e^{-x^{2} / 2}$ \\
1 & $\frac{3}{2}$ & $x e^{-x^{2} / 2}$ \\
2 & $\frac{5}{2}$ & $\left(x^{2}-\frac{1}{2}\right) e^{-x^{2} / 2}$ \\
3 & $\frac{7}{2}$ & $\left(x^{2}-\frac{3}{2}\right) x e^{-x^{2} / 2}$ \\
4 & $\frac{9}{2}$ & $\left(x^{4}-3 x^{2}+\frac{3}{2}\right) e^{-x^{2} / 2}$ \\
5 & $\frac{11}{2}$ & $\left(x^{4}-5 x^{2}+\frac{15}{4}\right) x e^{-x^{2} / 2}$ \\
\hline
\end{tabular}

It is quite clear from the table that the results when normalized agree with the well-known eigenvalues and eigenfunctions of quantum harmonic oscillator.

\section{Conclusion}

We have in this study presented a new framework for the determination of the Eigenvalues and Eigenfunctions of the Quantum Harmonic Oscillator. Starting with appropriate solutions of a Second Order Linear Differential Equation, we were able to recover the Schrödinger Equation of the Quantum Harmonic Oscillator by making use of Gram - Schmidt Orthogonalization process, which yielded two separate sequences of Even and Odd eigenfunctions. Explicit sample computations of the said eigenfunctions were also implemented. In a subsequent paper, we shall apply the framework developed here to a class of Quantum Anharmonic oscillators.

\section{References}

Bransden, B., \& Joachain, C. J. (2000). Quantum Mechanics.(2nd ed.).Pearson Education Limited, Asia.

Björck, $\dot{A}$. (1994). Numerics of Gram-Schmidt Orthogonalization Elsevier Science 655 Avenue of the Americas, New York, NY 10010.

Hendrik, F. H. (2004). Quantum Mechanics: A Conceptual Approach .John Wiley and Sons, Inc., Hoboken, New Jersey.

Ghatak, A., \& Lokanathan, S. (2012). Quantum Mechanics: Theory and Applications. (5th ed.). Trinity Press, India.

Griffiths, D. J. (1999). Introduction to Quantum Mechanics. Prentice Hall,Upper-Saddle River, New Jersey.

Miller, A. B. D. (2008). Quantum Mechanics for Scientists and Engineers. Cambridge University Press, New York. https://doi.org/10.1017/CBO9780511813962

Mendelson, E. (2004). Schaum's Solved Problems Series :3000 Solved Problems in Calculus. Tata McGraw-Hill.

Schaden, M. (2002). Quantum Finance. Physica A: Statistical Mechanics and its Applications.

Spiegel, R. M. (1976). Schaum's Outline of Theory and Problems of Advanced Mathematics for Engineering and Scientists. Schaum's Outline Series McGraw - Hill Companies, New York.

Wolf, R. (2021). Quantum Computing: Lecture Notes. University of Amsterdam. http://www.shoup.net/papers/dbounds.1

\section{Copyrights}

Copyright for this article is retained by the author(s), with first publication rights granted to the journal.

This is an open-access article distributed under the terms and conditions of the Creative Commons Attribution license (http://creativecommons.org/licenses/by/4.0/). 